\title{
DAS SCHAFFEN VON GEROLD TIETZ - LITERARISCHES ENGAGEMENT EINES VERTRIEBENEN SUDETENDEUTSCHEN
}

Gerold Tietz wurde 1941 in Horka (Nordböhmen) in einer sudetendeutschen Familie geboren. In dem Dorf lebten neben Deutschen auch Tschechen, Roma und Juden. In der Familie gab es auch tschechische Verwandten und etliche von den deutschen sprachen gut tschechisch und pflegten Kontakte mit den tschechischen Kulturkreisen.

Nach dem Krieg wurde er mit seiner Familie nach Schwaben vertrieben. Tietz studierte Geschichte, Französisch und Politik. Seit dem Jahre 1969 lebte der promovierte Historiker in Esslingen, wo er dreißig Jahre am Gymnasium unterrichtete.

In den autobiographisch geprägten Romanen Böhmische Fuge (1997), Böhmisches Richtfest (2007) und in Böhmische Grätschen (2009) versucht Tietz sowohl die offiziellen gesellschaftlich-politischen Ereignisse mit bekannten politischen und künstlerischen Persönlichkeiten, als auch die Episoden aus dem Bereich des Alltags der „kleinen Leute“, die sich mit den Konsequenzen der geschichtlichen Wenden für ihr persönliches Leben auseinandersetzen müssen, zu behandeln.

Im Beitrag werden die Umstände der Koexistenz der Deutschen und Tschechen untersucht und mit dem authentischen geschichtlichen Hintergrund konfrontiert. Dabei werden bei diesen Ethnien die negativen Erscheinungen nicht übersehen und die positiven als Beitrag für die heutige europäische Multikulturalität präsentiert.

Sudetedeutsche, Zweiter Weltkrieg, tschechisch-deutsche Beziehungen, Vertreibung, Autobiographie

Gerold Tietz lernte ich im Jahre 2005 bei seiner Lesung in Olmütz kennen. Ich las dann sein Romandebüt aus dem Jahre 1997 Böhmische Fuge. Davor erschien im Jahre 1989 ein Sammelband mit den satirischen Texten Satiralien, Berichte aus Beerdita, in dem der Autor seinen spießigen Mitbürgern einen Spiegel vorhält. Mit

\footnotetext{
${ }^{*}$ Mgr Jan Kubica, Ph.D., Pädagogische Fakultät der Palacky Universität in Olomouc, Institut für Fremdsprachen, deutsche Sektion, Zizkovo nam. 5, 771 40, Olomouc, Tschechien. E-mail: jan. kubica@upol.cz
} 
diesem Sammelband zeigte Tietz offensichtlich sein bürgerliches Engagement mit Hilfe von Scharfsinn und Humor. In dem Romandebüt sowie in den weiteren, überwiegend autobiographisch geprägten Romanen beruht, meines Erachtens, ebenfalls eine besondere Art von dem literarischen Engagement in Bezug auf die Geschichte des Zweiten Weltkrieges und auf die darauffolgende tragische Konsequenz - die Vertreibung der Sudetendeutschen, die er als kleines Kind erlebte. Gerold Tietz war Historiker und Politologe und versuchte immer fundiert in seinen autobiographischen Romanen den Einfluss der Ereignisse der sog. „Großen Geschichte“ auf das Leben der sog. „kleinen Menschen“ objektiv zu kontextualisieren. In diesem Beitrag wird versucht das Herantreten des Autors an die Problematik seiner Familiengeschichte mittels Vermittlung seiner Kindheitserinnerungen sowie Erinnerungen seiner Vorfahren in dem Zusammenhang mit der Theorie von Aleida Assman Drei Formen von Gedächtnis (Assman 2006, S. 16-17) und mit Hilfe des Begriffs von postmemory von Marianne Hirsch zu untersuchen. (Hirsch 2008, S. 105-106) Das literarische Schaffen von Gerold Tietz thematisiert die problematische Geschichte der deutsch-tschechischen Beziehungen und dieses Thema scheint in dem literarischen Engagement von Gerold Tietz richtungsweisend zu sein.

Der erste Roman von Tietz ist zum Teil autobiographisch geprägt und stellt das Leben einer sudetendeutschen Familie in Horka in Nordböhmen in den 1930er und 1940er Jahren, die Vertreibung aus diesen Gebieten und die Integration der Vertriebenen in Deutschland dar. Der Autor thematisiert darüber hinaus auch das Zusammenleben von Tschechen, Deutschen, Juden und Roma in der Tschechoslowakei. Er identifiziert sich am meisten mit der Figur Gernots, dem Sohn der Familie in Horka. (Kubica 2016, S. 250) Der Autor äußert sich dazu in einem Interview aus dem Jahre 2007. (Bachoríková, Jahodová 2007, S. 23)

Gerold Tietz wurde wie seine Figur Gernot 1941 in Horka (tsch. Horky u Dubé) in Nordböhmen in einer sudetendeutschen Familie geboren. In Horka lebten auch Tschechen, Juden und Roma. Die Familie Tietz hatte tschechische Verwandte, wobei einige aus dem deutschen Teil der Familie auch Tschechisch sprachen und das tschechische Kulturleben aktiv verfolgten. (Kubica 2016, S. 251)

Hier bietet sich an, auf das am Ende dieses Beitrags angeführte Zitat von Tietz über seine Position ,zwischen zwei Stühlen“ (Maier 2009, S. 1) hinzuweisen. Diese Position gilt, meines Erachtens, für den Autor ebenfalls in Bezug auf die deutsch-tschechischen Beziehungen. Er kann sich zum Teil mit seinen tschechischen Verwandten identifizieren, mit denen er seit den 1960er Jahren intensive Kontakte pflegte, indem er sich ihre Familiengeschichte erzählen ließ. Als Sudetendeutscher kann er sich mit den Deutschen in Nachkriegsdeutschland auch nur zum Teil identifizieren und bewahrt sich dadurch einen Abstand - eine Position „Zwischen zwei Stühlen“. (Maier 2009, S. 2)

Nach dem Kriegsende wurde die Familie vertrieben und ließ sich in Bayern nieder. Tietz besuchte das Gymnasium in Tuttlingen und studierte an den Universitäten in Tübingen, Berlin und Paris Geschichte, Französisch und Politologie. 
Im Jahre 1969 promovierte er im Fach Historiographie und unterrichtete Deutsch, Geschichte und Politik am Gymnasium in Wendlingen. Ab 1969 lebte er mit seiner Frau, der Schriftstellerin Anne Birk, in Esslingen. Im Jahre 2007 wurde der Autor mit dem Sudetendeutschen Kulturpreis für Literatur ausgezeichnet. Gerold Tietz starb 2009 in Esslingen. (Maier 2009, S. 1)

Die Idee, Tietz' Romandebüt als Böhmische Fuge zu betiteln, hatte der Verleger von Tietz - dieser Titel soll die kulturelle Mehrstimmigkeit der Figurenwelt, welche aus Tschechen, Juden und Deutschen besteht, zum Ausdruck bringen. (Bachoríková, Jahodová 2007, S. 23)

Der Autor schreibt dazu:

Eine Mehrstimmigkeit, die sicherlich nicht immer reibungslos war - da gab es Spannungen, da gab es Schwierigkeiten, aber es war letztlich ein jahrhundertelanges Zusammenleben. Von daher ist eine Trennung in tschechische, jüdische und deutsche Kultur eigentlich sehr einseitig, diese Kulturen hingen immer sehr stark zusammen. Und die tschechischen Historiker heute sind, soweit ich weiß, dabei, zum Beispiel auch Deutsche wieder in ihre eigene Kultur zurückzuholen. Wie hoffentlich auch Sudetendeutsche dabei sind, ihre tschechischen Nachbarn und ihre jüdischen Freunde wieder in ihre Geschichten und in ihr Gedächtnis zurückzuholen. (Bachoríková, Jahodová 2007, S. 23)

Bachoríková und Jahodová bezeichnen den Roman als literarische Collage, in welcher der auktoriale Erzähler Gernot als eine Stimme der Fuge einen fiktiven Dialog mit Hašeks Schwejk führt und dabei diverse geschichtliche Perioden der tschechischen und der deutschen bzw. der tschechisch-deutschen Geschichte kommentiert. Tietz erwähnt die Schlacht bei Königsgrätz und historische Persönlichkeiten wie Karel Hynek Mácha, Karel Čapek, Lída Baarová und Joseph Goebbels. (Bachoríková, Jahodová 2007, S. 23)

Bevor die nationalsozialistischen Maßnahmen in den sudetendeutschen Gebieten in Kraft traten, herrschte in dem Heimatdorf von Tietz Horka eine durchaus multikulturelle Atmosphäre:

Hier in Horka lebte man jedenfalls zusammen. Man hatte einen ungarischen Kutscher, slowakische Dienstmädchen, einen Prager Studenten, der beim Dorfschullehrer in den Ferien Deutsch lernte. Den schwarzen Hengst im Stall hatte sein Vater einem Zigeuner abgekauft und der Grünhopfen, der ging an die jüdischen Händler in Dauba, die ihn an alle Welt verkauften. (Tietz 2009, S. 130)

Diese Situation veränderte sich im Jahre 1938 schnell. Dies zeigt der Autor der Böhmischen Fuge an der Figur Gustavs, der Schwiegervater von Gernots Mutter Anna war. Als Belohnung für seine Verdienste in den Kämpfen gegen die tschechischen Partisanen im September 1938 - er war Kommandant einer SATruppe - bekam er einen ,Reichsmusterhof' in Horka, wo er im Rahmen von Zwangsarbeit sogenannte Ostarbeiter einsetzen durfte. Später wurde er vom Gauleiter zur Verwaltung der Gemeinde aufgefordert. (Tietz 2005, S. 58-59) 
Die Auswirkungen der nationalsozialistischen Ideologie schlugen sich auch im Schulbetrieb nieder. Tietz zeigt das in seinem Roman folgendermaßen:

In dem Jahre [1938], als in Dresden die NS-Volkswohlfahrt beginnt, Volksgasmasken zu verteilen und in Eger Wallenstein bei den Festspielen mit großem Pomp ermordet wird, bringen die Schulkinder aus Binai von ihrem Ausflug in die Sächsische Schweiz Nazizuckerl, aber nicht nur himbeerrote Bonbons mit schwarzen Hakenkreuzln, sondern auch Bilderbögen zum Ausschneiden mit Helm und Adler, Säbel und Totenkopf und ein Gesellschaftsspiel, das sich überall lustig anpreist und den Titel trägt ,Juden raus‘. (Tietz 2005, S. 49)

Im Juli 1945 wechseln sich in Horka Sowjets, tschechische Partisanen und Soldaten der tschechoslowakischen Armee Ludvík Svobodas ab. Dank der rechtzeitigen Warnung der Dienstmagd kann sich Anna vor den sowjetischen Soldaten verstecken, die sonst alle Frauen aus der Gemeinde ins Unbekannte abführen. Gustav, der Besitzer des ,Reichsmusterhofes ' wird von den Partisanen in ein Sammellager in Böhmisch Leipa deportiert; Gernots Mutter Anna wird von tschechischen Gendarmen aufgefordert, möglichst schnell nach Deutschland auszureisen. Sie packt das Nötigste und macht sich mit Gernot, der damals vier Jahre alt war, seinem jüngeren Bruder Hagen und Tante Rosa auf den Weg. (Tietz 2005, S. 86-93)

Zuerst zu Fuß, dann mit einem Pferdefuhrwerk, anschließend in einem überfüllten Zug kommen sie nach Böhmisch Leipa in das Internierungslager. ${ }^{1}$ Auf dem Bahnhof betrachtet Anna die von Prag kommenden Tschechen, die wie Touristen aussehen: Alle tragen auffällig viele leere Rucksäcke und Taschen. Es sind die sogenannten ,Goldgräber', also Plünderer, die auf den Augenblick warten, in dem die Deutschen ihre Häuser verlassen. (Tietz 2005, S. 95)

Im Lager in Böhmisch Leipa verbringt Anna mit der Familie zwei Tage und vor der Gewalt der Sowjets rettet sie sich, indem sie ihr Gesicht hinter einem Tuch versteckt und mit Holzkohle beschmiert. Sie werden mit dem Fuhrwerk bis zur sächsischen Grenze gefahren und müssen zu Fuß weitergehen. Nach drei Tagen, an denen sie herumirren und sich vor der Verfolgung durch scharf schießende sowjetische Soldaten verstecken müssen, erreichen sie endlich Bad Schandau. (Tietz 2005, S. 97-99)

Nach den Feststellungen von Arburg und Staněk überlebte eigentlich bis heute ein Mythos, dass an der wilden Vertreibung vor allem die Anhänger der Revolutionsgarden $(\mathrm{RG})$ teilgenommen haben. Die ersten wilden Vertreibungen wurden durch die Partisanentruppen nach den Direktiven des Generalstabs des Nationalen Verteidigungsministeriums durchgeführt. Bald hat diese Aufgabe die Tschecho-

${ }^{1}$ Der Internierungslager in Böhmisch Leipa (tsch. Česká Lípa) wurde für die Deutschen aus der breiten Umgebung im Juni 1945 errichtet. Ab Mitte Juli kam es zu Vertreibungen der Deutschen und zu Wohnungsplünderungen durch das 28. Regiment der Tschechoslowakischen Armee unter Führung des Oberstleutnants František Voves. Aus Böhmisch Leipa und aus der Umgebung wurden im Sommer 1945 ungefähr 18500 Personen vertrieben und es kam wenigstens zu 80 Selbstmorden (Padevět 2016, S. 355). 
slowakische Armee übernommen. Die Sowjetische Armee hat sich an den Vertreibungen nur selten beteiligt. (Arburg, Staněk 2010, S. 97-99).

Bemerkenswert ist ebenfalls die Feststellung von Detlef Brandes bezüglich der Gewalt der sich an der Vertreibung beteiligten tschechoslowakischen Subjekte. Er berichtet über die Stellungnahme von Molotov zu Durchführungsmethoden der Vertreibung in der Tschechoslowakei. Molotov soll vor viel zu schnellem und hartem Vorgehen gewarnt haben. Er sei auch gegen die Bezeichnung von Deutschen (weiße Armbinde mit dem Buchstaben N) gewesen. Die drastischen Maßnahmen sollten nur die aktiven Hitleranhänger betreffen und eben nicht diejenigen, die sich während des Krieges passiv verhielten. (Brandes 2002, S. 333)

Nach Arburg und Staněk kam bei den Fußmärschen in Richtung Bad Schandau zu vielen Todesfällen und zum psychischen und physischen Foltern. Dramatisch waren ebenfalls die Zugtransporte, die überwiegend in Pirna oder in Bad Schandau endeten. Die Frachtzüge wurden meistens aus den dachlosen Waggons zusammengestellt. (Arburg, Staněk 2010, S. 127-128)

Tietz reichert die spannenden Romanepisoden meistens mit Schilderung von zeitlich parallel verlaufenden historischen Ereignissen an. (Kubica 2016, S. 258)

Noch ahnte Anna nicht, dass sich die Herren in Potsdam auf das Wort, Überführung einigten. ,Überführung ... in ordnungsgemäßer und humaner Weise‘. Etwa zur selben Zeit explodierte ein Munitionslager in Schönpriesen, einem Vorort von Aussig. Tschechische Soldaten des 28. Regiments unter dem Obersten Voves trieben daraufhin die deutsche Zivilbevölkerung von Aussig auf der Elbebrücke zusammen und stürzten viele Hunderte in den Fluss. Aus dem Krematorium des von der Roten Armee befreiten KZ Theresienstadt stieg ein letztes Mal Rauch auf. Die Toten des Massakers von Aussig, die nicht elbeabwärts schwammen, wurden hier verbrannt. (Tietz 2005, S. 96)

Obwohl der Autor die Vertreibung als ein wenige Jahre altes Kind erlebte, behielt er seine Erinnerungen an diese dramatische Zeit:

Es gibt sicherlich ganz kritische Punkte. Zum Beispiel, wenn ich über das Thema Flucht und Vertreibung schreibe, da kommt dann schon ein Stück der eigenen Erinnerung hoch. Es gibt da zum Beispiel die Szene, wo ich in Altmarkt [Altenmarkt] in Bayern vom Wagen wie ein Stück Gepäck heruntergeworfen wurde. Hingeworfen an einen Ort, von dem man wusste, dass man dort nicht hingehört und dass einen dort auch niemand will. (Bachoríková, Jahodová 2007, S. 24)

Tietz hat diese Problematik zwar mehrfach in seinen Büchern thematisiert, als Historiker recherchiert, in Diskussionen und in Interviews besprochen, trotzdem empfand er diese frühe Kindheitserfahrung ziemlich lange als Trauma:

Ja, ein Beispiel dazu wäre dieser Zwang, mich ständig umzudrehen, den ich eine Zeitlang hatte. Der stammt sicherlich aus diesen Tagen der Flucht. Das äußerte sich etwa darin, dass ich aus keinem Fenster sehen konnte, weil ich immer dachte, hinter mir steht einer und wirft mich herunter. Also eine Zwangsvorstellung, ein Beispiel für verschiedene Traumata. (Bachoríková, Jahodová 2007, S. 24) 
Die Problematik der Vertreibung in dem Zusammenhang mit dem Nationalsozialismus und mit dem Zweiten Weltkrieg wird ebenfalls in der Gegenwart in Tschechien ziemlich kontrovers wahrgenommen. Vor allem bei den Kommunisten und bei der älteren Generation ist immer noch die Überzeugung von der kollektiven Schuld der Deutschen an dem Zweiten Weltkrieg aktuell. Michaela Peroutková spricht von der Dichotomie der Täter und der Opfer, in dem Sinne, dass die Tschechen in ihrer geschichtlichen Interpretation nach dem Zweiten Weltkrieg ein Bild von den Tätern, die nur Deutsche waren und von den Opfern, die nur Tschechen waren, geschaffen haben. Diese Dichotomie war den Sudetendeutschen natürlich unverständlich, denn es gab Täter und Opfer auf beiden Seiten. Es gab auch fast keine Differenzierung zwischen den aktiven Nationalsozialisten und den deutschen Antifaschisten. Es kam eigentlich ebenfalls zu keiner konsequenten Bestrafung von tschechischen Kollaborateuren und von den Anhängern der tschechoslowakischen faschistischen Strukturen. Oft wurden nämlich nach dem Kriegsende aus den tschechischen Kollaborateuren Anhänger der Revolutionsgarden und Kommunisten, die sich an den Exzessen während der Vertreibung der Sudetendeutsch intensiv beteiligt haben. Es wird ebenfalls die Tatsache marginalisiert, dass im Holocaust nicht nur die tschechischen sondern auch die deutschen Juden gelitten haben. Die deutschsprachigen Juden, die die Konzentrationslager überlebten und in die Tschechoslowakei zurückkehrten, wurden als Sudetendeutsche vertrieben. (Peroutková 2008, S. 100-103)

Ebenfalls problematisch ist eine der Konsequenzen des tschechischen Nachkriegsnationalismus, der nicht nur die Kommunisten sondern das ganze politische Parteienspektrum betraf. Es ist die Behauptung, dass die Tschechen am meisten unter der deutschen Diktatur litten. Es wurde jedoch nachgewiesen, dass es vor allem die tschechischen und die deutschen Juden, Roma und die politischen Gegner abgesehen von der Nationalität waren, die Opfer des Naziregimes waren. (Peroutková 2008, S. 56)

Auf der deutschen Seite ist die Situation nicht viel besser. Von Bad Schandau fährt Anna mit der Familie auf einem Dampfer nach Dresden. Mit dem Zug sollen sie weiter nach Hof gelangen. Vor Plauen ist die Bahnstrecke wegen einer zerstörten Brücke unterbrochen, und sie müssen sich unverhofft in der sowjetischen Besatzungszone aufhalten. Sie kommen zum Glück mit einem Fuhrwerk in die amerikanische Zone, nach Hof, aber diese Fahrt muss Anna einem deutschen Bauern mit einem goldenen Ring bezahlen. Im Sammellager in Hof kann die Familie Rast machen und sich genügend verpflegen. Mit den immer neu ankommenden Vertriebenen wird die Lebensmittelzuteilung von Tag zu Tag kleiner, deswegen machen sie sich wieder auf den Weg: über Nürnberg geht es nach Simmelreith bei Traunstein, wo ein Frontkamerad von Annas Mann ein Haus hat. (Tietz 2005, S. 100-105)

Im weiteren Teil des Romans befasst sich Tietz mit der tatsächlich problematischen Integration der deutschen bzw. sudetendeutschen Vertriebenen und Flüchtlinge in Deutschland. Nach den tragischen und traumatischen Erfahrungen, 
die sie erst kurz vorher, während ihrer Vertreibung aus der Tschechoslowakei bzw. aus anderen osteuropäischen Ländern, erleben mussten, sahen sie sich mit den recht großen Antipathien vieler Mitbürger und Verwaltungsbehörden konfrontiert.

Das Haus des Frontkameraden in Simmelreith ist überfüllt - die vierköpfige Familie bekommt ein kleines Zimmer mit schimmliger Decke zugeteilt. Sie brauchen Essensmarken und Bezugsscheine für weitere Gebrauchswaren, deshalb meldet sich Anna beim Ortsverwaltungsamt an, wo sie aber weder auf Verständnis noch auf Sympathie trifft:

Wen will denn Frau Pohlding noch aufnehmen, ihr Haus ist doch vollgestopft mit entlassenen Soldaten. Höchste Zeit, dass Herr Pohlding zurückkommt. [...] Und was ist mit Ihren Eltern? Die werden doch auch bald der Gemeinde zur Last fallen. (Tietz 2005, S. 114)

Auch der Arbeitgeber, bei welchem Anna eine schwere körperliche Arbeit verrichten muss, ist gefühllos und arrogant ihr gegenüber:

Als Anna den Lohn entgegennahm, erklärte sie dem Bauern, dass sie nicht fortsetzen könne, da ihre Arme ganz angeschwollen seien. Der Reinhuber entgegnete, er habe Gesinde genug, um die Kartoffelernte einzubringen. Und dann fragte er Anna unverblümt, ob er nicht einmal bei ihr zu Hause vorbeikommen könne, am besten, wenn die Kinder nicht da wären. (Tietz 2005, S. 117)

Ein weiteres Problem ist für Anna die Integration, denn infolge der Entnazifizierung darf sie den Lehrerberuf nicht ausüben. Sie ist studierte Lehrerin, die in Böhmen in einer Klosterschule unterrichtete und von dort eine vorzügliche Empfehlung bekommen hat. Sie bewirbt sich beim Schulrat um eine Stelle. Beim Bewerbungsgespräch erwähnt Anna ihre Mitgliedschaft in der NS-Frauenschaft, wo sie jedoch nicht aktiv tätig war - von ihrer Teilnahme am Erntedankfest in Böhmisch Leipa abgesehen. Der Schulrat muss aber dem strengen Verbot der Anstellung von Lehrern, die Mitglieder einer NS-Organisation waren, folgen. (Tietz 2005, S. 115)

Den nachfolgenden Roman von Tietz, das Böhmische Richtfest aus dem Jahr 2007 können wir als Fortsetzung seines Romandebüts betrachten. Es taucht hier wieder Gernot als Hauptfigur auf, der mit den Augen eines Kindes die Vertreibung verfolgt. Der größte Teil des Textes wird aber der Problematik der Integration der Sudetendeutschen in die westdeutsche Gesellschaft gewidmet. Der Roman endet mit der Okkupation der Tschechoslowakei durch fünf Armeen des Warschauer Paktes im Jahre 1968. (Kubica 2016, S. 251)

Im Folgenden soll auf drei Episoden aus diesem Buch eingegangen werden, in denen es um die sudetendeutschen Antifaschisten, das Zusammenleben der Vertriebenenkinder mit ihren Mitschülern und die problematische Identitätssuche der Sudetendeutschen in Deutschland in der Nachkriegszeit geht. Diese Episoden können wir als repräsentative Beispiele des literarischen Engagements bei Gerold 
Tietz betrachten. Die erste zeigt recht anschaulich die antifaschistische Bewegung der Sudetendeutschen in Nordböhmen.

Tietz schildert in diesem Roman den in Horka lebenden sudetendeutschen Antifaschisten Grasselt, der offen gegen die Henleinbewegung protestiert und den Hitlergruß ablehnt. Während des Krieges wird er von seinen Mitbürgern als „bolschewistischer Hund" beschimpft; nach dem Krieg wird er wie alle anderen Sudetendeutschen von den Tschechen „Nazischwein“ genannt und ebenfalls vertrieben. (Tietz 2007, S. 119-121)

Wie sich aus Zdeněk Radvanovskýs Recherchen ergibt, hatte die antifaschistische Widerstandsbewegung in den Grenzgebieten keine zentrale Führung. Jedoch seien in Nordböhmen Aktivitäten einiger Widerstandsgruppen auf dem Gebiet von Tetschen-Bodenbach, Dux, Teplitz und Brüx dokumentiert worden. Als die bedeutendste Gruppe betrachtet Radvanovský die antifaschistische Jugend aus dem Gebiet von Teplitz unter der Führung von Herta Lindner. Diese Gruppe präsentierte sich offiziell als Bergsteigerverein und führte bereits vor dem Krieg deutsche Antifaschisten über die Grenze. Die Mitglieder der Gruppe schrieben während des Krieges Flugblätter, übersetzten und lieferten sie nach Dresden. Die Gruppe wurde im Herbst 1941 ermittelt, neununddreißig Mitglieder wurden verhaftet und Herta Lindner wurde im Januar 1943 in Berlin-Plötzensee hingerichtet. (Radvanovský 1999, S. 34-35)

Zum Widerstand in Nordböhmen äußert sich Radvanovský ebenfalls:

Gegen den aufkommenden Nationalsozialismus stellten sich jedoch in Nordwestböhmen nicht nur tschechische und deutsche Kommunisten und Sozialdemokraten, sondern auch tschechoslowakische Staatsbürger jüdischer Herkunft. (Radvanovský 1999, S. 37)

Wie oben erwähnt, thematisiert Tietz in seinem Roman-Debüt das Zusammenleben von Tschechen, Deutschen, Roma und Juden in Nordböhmen. Nach Radvanovský existierte in dieser Region ebenfalls die antifaschistische Bewegung der jüdischen Bevölkerung. Als Vertreter des jüdischen Widerstands erwähnt Radvanovský den aus Aussig stammenden Maler Ernst Neuschul, einen Absolventen der Prager Kunstakademie, der Kontakte zu Franz Kafka und Max Brod pflegte. Er war Mitglied der Künstlergruppen ,Novembergruppe“ und ,Neue Sachlichkeit'. Anfang der 1930er Jahre war er Sympathisant der deutschen Kommunistischen Partei, deren Mitglied er aber nie wurde. Nach 1933 protestierte er mit seinen Werken gegen den Nationalsozialismus. (Radvanovský 1999, S. 37-38)

Die zweite Episode präsentiert eine andere Art des literarischen Engagements bei Gerold Tietz. Er weist deutlich auf das problematische Zusammenleben der sudetendeutschen Vertriebenen in ihrer neuen Heimat hin. Gernot trifft ähnlich wie seine Mutter bei Nachbarn und Behörden auf große Antipathien und auf Unverständnis seiner Mitschüler. Er wird als Flüchtling aus dem Osten mit unklarer Herkunft bezeichnet, ,Watzlaw‘ oder ,Polack‘ genannt. Mit der Zeit gewöhnt er 
sich daran, dass seine Schulkameraden am Gymnasium kein Interesse an seinen ,Flüchtlingsgeschichten“ haben und keine ,schlechten Geschichten aus dem Osten" (Tietz 2007, S. 105) hören wollen. Sie möchten sich viel lieber über Mode, Fahrräder und über die neuen Eissorten im örtlichen Café unterhalten. (Tietz 2007, S. 105)

In der dritten Episode kommt ein weiterer problematischer Aspekt der Integration der sudetendeutschen Vertriebenen in Nachkriegsdeutschland zum Ausdruck und zwar, wie oben erwähnt, die problematische Identitätssuche infolge ihres Heimatverlustes. Gernot begegnet diesem Problem, als ihn sein Lehrer nach seiner Herkunft fragt. Nach Wurmlingen ist er aus Bayern gekommen, und so überlegt er sich, ob er Bayern oder doch eher Böhmen sagen sollte. Aus Böhmen wurde er zwar vertrieben, aber er wurde dort auch geboren. Er zögert ziemlich lange und schließlich sagt er, was er von seiner Mutter hörte (Tietz 2007, S. 84-85): „Ich komme aus dem sechsten Kontinent - aus Böhmen.“ (Tietz 2007, S. 85)

Mit derselben Frage muss sich auch Anna befassen, als sie einen neuen Personalausweis beantragt. Sie wurde 1913 in Brozen in Böhmen in der Regierungszeit Kaiser Franz Josefs geboren. Als sie dann in Brozen zur Schule kam, wurde die Tschechoslowakei gegründet. Als verheiratete Frau lebte sie seit 1938 im Sudetenland, das nun dem Deutschen Reich angehörte. Im Sommer 1945 wurde Anna vertrieben, wobei ihr von der tschechoslowakischen Regierung ihre tschechoslowakische Staatsangehörigkeit aberkannt wurde - die Alliierten wollten ihre deutsche Staatszugehörigkeit nicht anerkennen. Wenn sie als ihr Herkunftsland die inzwischen kommunistische Tschechoslowakische Republik angeführt hätte, wäre sie von dem Beamten wohl für eine Kommunistin gehalten worden. Anna schweigt lieber und der Beamte lässt die Zeile zu Staatszugehörigkeit und Geburtsort frei. Der Staat, sei es Österreich-Ungarn, Tschechoslowakei oder Großdeutsches Reich war für sie immer etwas Unfassbares oder etwas Irreales gewesen - ein sechster Kontinent. (Tietz 2007, S. 201, 203-204)

Tietz' letzter Roman, die Böhmischen Grätschen aus dem Jahre 2009, ist ebenfalls autobiographisch geprägt. In einem gewissen Sinne handelt es sich um eine Art Fortsetzung der vorherigen zwei Romane Böhmische Fuge und Böhmisches Richtfest, die autobiographischen Züge tragen. Die Autofiktionalität hat bei Tietz eine besondere Form, denn er erlebte die Vertreibung als vierjähriges Kind. Authentisch bzw. autobiographisch ist in den zwei oben erwähnten Romanen die Erzähllinie der Vertreibungs- und Fluchtszenen. Eben die Selbstbezüge bei Tietz und zugleich seine Neigung, die persönliche Familiengeschichte historiographisch zu kontextualisieren, resultiert in seinem literarischen Engagement.

Auch in dem Roman Böhmische Grätschen steht im Mittelpunkt der Handlung die Figur Gernots, der seine Besuche als Erwachsener bei seinen Verwandten in Böhmen in den 1960er und 1990er Jahren beschreibt. Seine Begleiterin durch die Familiengeschichte ist seine tschechische Cousine Jana. 
In diesem Text thematisiert der Autor nicht nur wie bereits in den früheren Werken seine eigenen Erinnerungen, sondern auch die Eindrücke, Erinnerungen und diversen Familiengeschichten seiner tschechischen Vorfahren. Diesen Vorgang können wir als eine weitere Art der spezifischen Autofiktionalität bei Tietz betrachten. Seine Kindheitserinnerungen und ihre historischen Parallelen bereichert er durch die Erinnerungen seiner tschechischen Vorfahren. Es handelt sich vor allem um die Zeit der 1950er und 1960er Jahre.

Diese Art Vermittlung von historischen Erlebnissen und Erfahrungen können wir auf Grund der Theorie über Drei Formen von Gedächtnis von Aleida Assmann erklären. Bei Tietz handelt es sich um das individuelle Gedächtnis in Bezug auf seine Kindheitserinnerungen an die Zeit der Vertreibung. Das kommunikative Gedächtnis können wir auf die gemeinsamen Erinnerungen von dem Autor und von seiner Mutter bzw. mit seiner Tante, mit denen er die Vertreibung erlebte, beziehen. (Assman 2006, S. 16-17)

Die von Tietz dargestellten traumatischen Erfahrungen aus der Zeit der Vertreibung sind nicht allein dem Opfergedächtnis im Sinne Aleida Assmann zuzurechnen. In seinen Romanen werden sie zum Bestandteil vom kulturellen Gedächtnis, das oberhalb des kommunikativen und des kollektiven liegt. (Assman 2006, S. 21-25)

Mit dem Begriff postmemory (Hirsch 2008, S. 105-107) bezeichnet Marianne Hirsch die Vermittlung von historischen Erlebnissen und Erfahrungen. Kathy Behrendt weist bei Hirsch auf ihre spezifische Orientierung von postmemory in Bezug auf die Beziehung zwischen den Kindern und ihren Eltern, die Opfer oder Augenzeugen von einem Trauma wurden hin. ${ }^{2}$ (Behrendt 2013, S. 51) Bei Gerold Tietz handelt es sich um eine besondere Art von postmemory oder eher um eine Kombination von postmemory und von seinen eigenen Erinnerungen, denn er ließ sich von der Vertreibung von seiner Mutter und Tante erzählen und dabei hat er sich seine eigene traumatischen Erinnerungen an diese Zeit belebt. Behrendt betont ebenfalls, dass Gedächtnis selektiv ist und man kann nicht alle Details seiner Erfahrung hervorrufen. Die Wahl kann dann entweder gut oder schlecht sein. Jedoch bei den gut gewählten Erinnerungen können die gewählten Details der Vergangenheit signifikant und relevant von der Perspektive der Gegenwart sein und in diesem Sinne ist es richtungsweisend dafür, was wir von der Vergangenheit wissen, wie wir diese verstehen und wie wir sie wahrnehmen. (Behrendt 2013, S. 53). Dieses Prinzip vom Erinnern kommt, meines Erachtens, auch in den autobiographisch geprägten Romanen von Tietz zum Ausdruck. Die Wahrnehmung bzw. die Interpretation der Vergangenheit (der Geschichte) prägt bei dem Autor wesentlich seine Bildung (Historiographie und Politologie).

\footnotetext{
${ }^{2}$ Her specific focus in her initial work on postmemory was on the relationship that children have parents who were victims or witnesses of such trauma. (Behrendt 2013, S. 51)
} 
Veronika Tuckerová erwähnt diesen Begriff im Zusammenhang mit den traumatischen Erfahrungen der Mutter von Herta Müller in deren Roman Atemschau$\mathrm{kel}$. Dieses Prinzip steht, meines Erachtens, nah den von Tietz vermittelten Erinnerungen seiner Verwandten. (Tuckerová 2010, S. 27)

Gernot stellt fest, dass seine Familie einen recht multikulturellen Charakter hat:

,Eines mußt du dir merken', fuhr Jana fort, ,unsere Familien, waren immer schon gemischt. Deine Mutter, die Zborschilová, die ist als elfjähriges Mädchen auf ,Tausch' nach Doubrava ins Isergebirge gegangen, um Tschechisch zu lernen. Und der Onkel deines Großvaters, der Turnprofessor, der ist in Prag in einem tschechischen Gesangverein gewesen. Und deine Tante Rosa, die ist in der Böhmischen Brüdergemeinde gewesen und hat aus dem ,New Gsengbüchlein' gesungen, ein Liederbuch, das schon Luther kannte und das 1531 in Jungbunzlau erschienen ist. (Tietz 2009, S. 9)

Neben den in dem Zitat erwähnten Mitgliedern der tschechischen Verwandschaft von Tietz war es auch sein Onkel Bohumil, der ein begeisterter Anhänger des tschechischen Turnvereins „Sokol“ (Falke) war. Tietz betonte immer, dass die meisten Mitglieder seiner Familie, ob Tschechen oder Deutsche zweisprachig waren. Die tschechischen Verwandten lebten in Mladá Boleslav (dt. Jung Bunzlau) und die deutschen in Prag. Diese Prager Deutschen sangen jedoch in einem tschechischen Gesangsverein und interessierten sich intensiv für die tschechische Kultur. (Bachoríková, Jahodová 2007, S. 23)

Alexander Maier nennt Gerold Tietz in seinem Nekrolog ,einen unermüdlichen Brückenbauer und Ermutiger". Er zitiert dabei auch die Selbstbeschreibung des Schriftstellers: „Zwischen zwei Stühlen fühle ich mich wohl. Das ist für einen Schreibenden der angemessene Platz." (Maier 2009, S. 2) Diese Stellungnahme, wie am Anfang des Beitrags erwähnt, können wir bei Tietz als eine Art von Grenzposition betrachten oder eher als eine Art von Gratwanderung zwischen der persönlichen Familiengeschichte, deren traumatischer Bestandteil die Vertreibung war, und der Geschichte Europas in der ersten Hälfte des 20. Jahrhunderts. Meier schreibt dem Autor der Böhmischen Fuge mit Recht die Rolle des Vermittlers zu: des Vermittlers zwischen den vertriebenen Sudetendeutschen und den Deutschen im Nachkriegsdeutschland.

Tietz' Schaffen können wir auch, wie oben erwähnt, als kulturelles Gedächtnis (Assman 2006, S. 23-25) betrachten. In diesem Zusammenhang wird sinnvoll Aleida Assmann zu zitieren:

Wie das kollektive Gedächtnis wird das kulturelle Gedächtnis gebraucht, um Erfahrungen und Wissen über die Generationenschwellen zu transportieren und damit ein soziales Langzeitgedächtnis auszubilden. Während jedoch das kollektive Gedächtnis diese Stabilisierung durch radikale inhaltliche Engführung, hohe symbolische Intensität und starke psychische Affektivität erreicht, stützt sich das kulturelle Gedächtnis auf externe Medien und Institutionen. Hier spielt die Auslagerung von Erfahrungen, Erinnerungen und Wissen auf Datenträger wie Schrift und Bild eine Entscheidende Rolle. (Assman 2006, S. 24) 
Wie aus dem Zitat hervorgeht, schuf Gerold Tietz, meines Erachtens, mit seinem Prosawerk einen wesentlichen Beitrag zur Vermittlung von Erfahrungen und Wissen seiner Generation und der Generation seiner Eltern- und Großeltern über die erste Hälfte des 20. Jahrhunderts, dessen geschichtliche Prozesse das Leben der Menschen nicht nur in Europa, sondern in der ganzen Welt oft tragisch und dramatisch prägten. In diesem Sinne ist es sehr wichtig dieses Wissen für die weiteren Generationen als Mahnung zu bewahren, zu dokumentieren und zu pflegen. In diesem Beitrag beruht das literarische Engagement von Gerold Tietz.

Das Schaffen von Gerold Tietz ist nicht so bekannt und kaum so anerkannt wie das Werk von Ota Filip und von Horst Bienek. Meiner Meinung nach verbindet alle drei Autoren nicht nur die Problematik des Heimatverlustes bzw. der Vertreibung, sondern auch das Herantreten an die literarische Bearbeitung dieser Problematik. Alle drei versuchen das aktuelle Geschehen der 1930er und der 1940er Jahre mit dem tieferen historischen Hintergrund zu kontextualisieren. Alle drei thematisieren ihre Autobiographien bzw. ihre Familiengeschichten in Bezug auf die Ereignisse der Zeit vor, während und nach dem Zweiten Weltkrieg - anders gesagt - sie zeigen in ihren Werken, wie die ,große Geschichte“ das Leben der „,kleinen Menschen“ prägt. Alle drei Autoren können wir als Vermittler, Brückenbauer und Gratwanderer zwischen Menschen verschiedener Nationen, Kulturen und Herkunft betrachten. Eben in ihrer Vermittlerfunktion beruht ihr gesellschaftliches Engagement. Solche Autoren werden auch heute gebraucht nicht nur in Europa, sondern in der ganzen Welt.

\section{LITERATURVERZEICHNIS}

Arburg A. von, Staněk T. (Hrsg.) (2010), Vysídlení Němců a proměny českého pohraničí 1945-1951. Duben - srpen/záři 1945: „Divoký odsun “ a počátky osidlování, SUSA, Středokluky.

Assman A. (2002), Drei Formen von Gedächtnis. In: P. Rautmann, N. Schalz (Hrsg.), Zukunft und Erinnerung. Perspektiven von Kunst und Musik an der Jahrtausendwende, Hauschild, Bremen, S. 14-26.

Bachoríková K., Jahodová L. (2007), Anne Birk und Gerold Tietz in Olmütz. Interview. Neuerscheinungen. In: B. Gunsenheimer (Hrsg.), Germling. Germanisten für Germanisten, Sommersemester 2007, Olomouc, S. 22-27.

Behrendt K. (2013), Hirsch, Sebald, and the Uses and Limits of Postmemory. In: The Memory Effects: The Remediation of Memory in Literature and Film, Wilfrid Laurier University Press, Waterloo, S. 51-67, https://scholars.wlu.ca/cgi/viewcontent.cgi?article=1008\&context=phil_faculty (stand: 17.01.2019).

Brandes D. (2002), Cesta k vyhnání 1938-1945. Plány a rozhodnutí o „transferu“ Nèmců z Československa a z Polska, Prostor, Praha.

Hirsch M. (2008), The Generation of Postmemory, „Poetics Today”, Bd. 29, Nr. 1, S. 103-128, http://urokiistorii.ru/sites/all/files/hirsch_generation_of_postmemory.pdf (stand: 02.06.2018).

Kubica J. (2016), Život ve třicátých a čtyřicátých letech dvacátého století v multikulturnich regionech středni Evropy na základě autobiografických próz německy pišicich autorů - Ota Filip, Horst Bienek a dalších, Univerzita Palackého v Olomouci, Olomouc. 
Maier A. (2009), Ein unermüdlicher Brückenbauer, http://www.geroldtietz.de/nachrufe.php (stand: 19.07.2017).

Padevět J. (2016), Krvavé léto 1945. Poválečné násili v českých zemích, Academia, Praha.

Peroutková M. (2008), Vyhnání. Jeho obraz v české a německé literatuře, Libri, Praha.

Radvanovský Z. (1999), Deutsche Antifaschisten in Nordwestböhmen. In: B. Bolzano Stiftung (Hrsg.), Unbekannte Schicksale. Internationales Seminar in Liberec (Reichenberg), Oktober 1997, Praha, S. 32-42.

Tietz G. (2005), Böhmische Fuge, Info-Verlag, Karlsruhe.

Tietz G. (2007), Böhmisches Richtfest, Stutz, Passau.

Tietz G. (2009), Böhmische Grätschen, Stutz Verlag, Passau.

Tuckerová V. (2010), Traumata Herty Müllerové, „Lidové noviny” 26.06, S. 27.

Jan Kubica

\title{
LITERARY WORK BY GEROLD TIETZ - LITERARY ENGAGEMENT OF AN EXPELLED GERMAN
}

\author{
(Summary)
}

Gerold Tietz was born in 1941 in Horka (north Bohemia) in a family of Sudeten Germans. Germans lived in this village together with Czechs, Roma people and Jews. The family also involved Czech relatives and many of German relatives spoke good Czech and kept relations with Czech cultural groups.

After the war Gerold Tietz and his family were expelled to Swabia. He studied history, French and political science. From 1969 the graduated historian lived in Esslingen where he taught in the grammar school for thirty years.

In the autobiographically oriented novels Böhmische Fuge (1997), Böhmisches Richtfest (2007) and in Böhmische Grätschen (2009) Tietz tried to depict official social-political events connected with famous political and cultural figures as well as the stories of ordinary days of "small people" who had to face the consequences of historic changes which influenced their lives.

The paper analyses the conditions of Czech and German coexistence and confronts the authentic historic context. Nevertheless, negative features of these ethnic groups are not overlooked and the positive ones are presented as a positive contribution to the current European multiculturalism.

Keywords: Sudeten Germans, the Second World War, Czech-German relations, expulsion, autobiography 